\title{
Évaluation surveillance de la rougeole, CSCom Central de Tominian, 2009 à 2018
}

\section{Measles surveillance assessment, Tominian Central Community Health Center, 2009 to 2018}

\author{
Togola OB ${ }^{1}$, Ballayira $Y^{2}$, Traoré $\mathrm{B}^{3}$, Kayembé Ken ${ }^{3}$, Sangho $\mathrm{O}^{4,5}$, N'Diaye $\mathrm{H}^{6}$, Ouattara $\mathrm{S}^{7}$, Yacouyé $\mathrm{H}^{8}$
}

1. Centre de Santé de Référence de Tominian, Ségou, Mali

2. Division Prévention et Lutte contre la Maladie, DGSHP, Bamako, Mali

3. Réseau Africain des Epidémiologistes de Terrain, AFENETMali, Bamako, Mali

4. DER des Sciences Biologiques et Médicales, FAPH/USTTB, Mali

5. Département d'Enseignement et de Recherche en Santé Publique, FMOS/USTTB, Mali

6. Direction Régionale de la Santé de Ségou, Mali

7. Direction Régionale de la Santé de Bamako, Mali

8. Direction Nationale des Services Vétérinaires, Bamako, Mali

Auteur correspondant : Dr Ousmane Boua Togola, Centre de Santé de Référence de Tominian, Ségou, 0022376366127 / 636677 09, ousmanebouatogola@gmail.com

\section{Résumé :}

Introduction : Au plan mondial, le nombre de cas de rougeole a augmenté de $300 \%$ lors des trois premiers mois de 2019 comparativement à la même période pour 2018. Les faibles couvertures vaccinales de l'aire de santé de Tominian Central, la notification de cas suspects par le district en 2018 et les récentes épidémies de rougeole survenue dans la région constituent une préoccupation sanitaire locale. Cette étude a pour but d'évaluer le système de surveillance de la rougeole de l'aire de santé de Tominian Central. Méthodes: Nous avons conduit une étude transversale utilisant les données de surveillance de la rougeole de 2009 à 2018 de l'aire de santé de Tominian Central. Nous avons appréciés les attributs d'organisation et de fonctionnement, utilité, sensibilité, réactivité, simplicité, sensibilité, représentativité et la qualité des données. Les agents chargés de la surveillance de la rougeole ont été interviewés. Résultats: À l'exception du cabinet médical, il existait un système de rapportage des données de la surveillance de la rougeole et la preuve de leurs transmissions au niveau supérieur est vérifiable. Le personnel du CSCom et du cabinet médical chargé de la notification n'ont réalisée aucune action de santé publique dans la surveillance de la rougeole. Un seul cas suspect de rougeole a été notifié par le Centre de santé de référence. Conclusion: Le système de surveillance de la rougeole de Tominian Central apparaît comme étant fonctionnel, moins utile, moins sensible, moins représentatif, moins réactif. Des efforts supplémentaires sont nécessaires pour le dynamiser.

Mots clés : évaluation, surveillance, rougeole, Tominian, Mali.

Abstract:

Introduction: Globally, the number of measles cases increased by $300 \%$ in the first three months of 2019 compared to the same period in 2018. Low immunization coverage in the Central Tominian Health Area, the reporting of suspected cases by the district in 2018 and recent measles outbreaks in the region are a local health concern. The purpose of this study is to evaluate the measles surveillance system in the Central Tominian Health Area. Methods: We conducted a cross-sectional study using measles surveillance data from 2009 to 2018 in the Central Tominian Health Area. We assessed the attributes of organization and functioning, usefulness, sensitivity, responsiveness, simplicity, sensitivity, representativeness and data quality. Measles surveillance officers were interviewed. Results: With the exception of the private health center, there was a system in place for reporting measles surveillance data, and evidence of transmission to the next level is verifiable. The CSCom and office staff responsible for reporting did not conduct any public health actions in measles surveillance. Only one suspected case of measles was notified by the Reference Health Centre. Conclusion: The measles surveillance system of Tominian Central appears to be functional, less useful, less sensitive, less representative, and less reactive. Additional efforts are needed to make it more dynamic.

Keywords: evaluation, surveillance, measles, Tominian, Mali.

Introduction :

La rougeole est fortement contagieuse et demeure l'une des principales causes de mortalité infanto-juvénile. Malgré la réduction considérable de la mortalité rougeoleuse, la couverture vaccinale avec le vaccin anti rougeoleux, la qualité des activités de vaccination anti rougeoleuse supplémentaires et la qualité de la surveillance de la maladie dans la région africaine n'ont pas encore atteint le niveau requis pour prévenir la résurgence de la rougeole (1). Au plan mondial, le nombre de cas déclarés a augmenté de $300 \%$ lors des trois premiers mois de 2019 par rapport à la même période pour 2018 (2). L'Organisation Mondiale de la Santé (OMS) estime que moins d'un cas sur 10 est déclaré dans le monde, avec des écarts entre les Régions $(2,3)$. Entre les semaines 1 et 14 de l'année 2019, 28487 cas suspects dont 107 décès ont été rapportés dans la zone CEDEAO (Communauté des Etats de l'Afrique de l'Ouest). Pendant la même période en 2018, 15267 cas suspects dont 85 décès ont été notifiés dans 14 Etats Membres de la CEDEAO dont le Mali (4). Au Mali, 1634 cas suspects de rougeole ont été enregistrés en 2018 parmi lesquels 1404 cas ont été 
prélevés et envoyés à l'Institut National de Santé Publique (INSP) dont 476 cas positifs et 4 décès chez des enfants de moins de cinq ans (5). Malgré, les efforts réalisés dans la vaccination, la rougeole demeure un problème de santé publique au Mali, à Ségou et à Tominian en particulier dans un contexte un marqué par l'insécurité et les crises sociales. En 2018, Ségou a notifié 93 cas suspects de rougeole dont 8 cas pour le district sanitaire de Tominian (6). Dans le cadre de la sécurité sanitaire mondiale, tous les états membres de l'Organisation Mondiale de la Santé (OMS) se sont engagés à atteindre une capacité de base pour la surveillance de santé publique (7). Les faibles couvertures vaccinales anti rougeoleuse de l'aire de santé de Tominian Central qui furent respectivement $77,84 \%, 77,04 \%, \quad 72,90 \%$ en $2016,2017,2018$, l'absence de rapport d'évaluation du système de surveillance de la rougeole de ladite aire de santé et les récentes épidémies de rougeole survenue dans d'autres districts de la région constituent une menace pour la sécurité sanitaire locale. La mise en œuvre d'une surveillance efficace est une condition fondamentale pour parvenir à éliminer la rougeole (10). Au regard de tout ce qui précède, il apparait important de savoir si le système de surveillance épidémiologique de la rougeole de l'aire de santé de Tominian Central du district sanitaire de Tominian est en mesure de répondre aux défis actuels ? L'objectif de la présente étude est d'évaluer le système de surveillance de la rougeole de l'aire de santé de Tominian Central du district sanitaire de Tominian sur la période de 2009-2018.

Matériel et méthodes :

Cadre de l'étude :

L'étude a été réalisée dans l'aire de santé de Tominian Central constituée de vingt-six villages pour une population de 25101 habitants. Le site d'étude choisie au hasard renferme en son sein un (1) cabinet médical privé et six sites d'agents de santé communautaire (ASC) qui participent tous aux activités de surveillances épidémiologiques.

Type et période d'étude :

Nous avons conduit une étude transversale du 1 er au 30 Juin 2019 en utilisant les données issues des activités de surveillance de la rougeole sur la période de 2009 à 2018 dans l'aire de santé de Tominian Central.

Population d'étude :

Les structures et personnel de santé exerçant sur l'aire de santé de Tominian Central et les chargés de la surveillance épidémiologique du CSRéf de Tominian et de la Direction Régionale de la Santé (DRS) de Ségou.

Techniques de collectes des données :

Les données ont été collectées par l'interview en administrant un questionnaire, par observation et revue documentaire à l'aide d'un formulaire d'extraction des données.

Outils de collectes des données :
La collecte a concerné les données liées à la surveillance de la rougeole au cours des dix dernières années. Les outils utilisés pour cette activité étaient le registre de notification des MADO (Maladie à Déclaration Obligatoire), les listes linéaires, les registres de surveillance épidémiologique du laboratoire du district. Une fiche d'enquête a été adressée aux personnes impliquées dans la surveillance de la rougeole du centre de santé communautaire de Tominian Central, du CSRéf et de la direction régionale de la santé de Ségou (DRS). Définitions opératoires :

Elles sont issues du guide d'évaluation de la surveillance élaboré par CDC (8).

Utilité: Un système de surveillance de la santé publique est utile lorsqu'il contribue à la prévention et au contrôle des événements négatifs liés à la santé, y compris une meilleure compréhension des implications de ces événements de santé publique. Un système de surveillance de la santé publique peut également être utile lorsqu'il permet de déterminer qu'un événement négatif lié à la santé précédemment considéré comme étant sans importance est réellement important.

Réactivité : Capacité du système à s'organiser, et à se déployer rapidement et efficacement pour la riposte.

Simplicité : La simplicité d'un système de surveillance de la santé publique se réfère à la fois à sa structure et à sa facilité d'utilisation. Les systèmes de surveillance doivent être les plus simples possible tout en atteignant leurs objectifs.

Sensibilité : La sensibilité d'un système de surveillance peut être considérée à deux niveaux. Tout d'abord, au niveau de la déclaration des cas, la sensibilité se réfère à la proportion des cas d'une maladie (ou tout autre problème de santé) détectés par le système de surveillance. Deuxièmement, la sensibilité peut se référer à la capacité de détecter les épidémies, y compris la possibilité de suivre l'évolution du nombre de cas au fil du temps.

Représentativité : Un système de surveillance de la santé publique qui est représentatif décrit avec précision, la survenance d'un problème de santé au fil du temps et sa distribution dans la population selon les lieux et les personnes.

Qualité des données : La qualité des données reflète l'exhaustivité et la validité des données enregistrées dans le système de surveillance de la santé publique.

Résultats :

Organisation et fonctionnement :

Notre étude a porté sur les structures de santé, le personnel impliqué dans la surveillance de la rougeole dans l'aire de santé de Tominian Central, les chargés surveillance du CSRéf et de la DRS de Ségou. L'aire de santé de Tominian Central, a un système de surveillance basé sur la notification des maladies à déclaration obligatoire. Dans l'aire de santé de Tominian Central, la surveillance de la rougeole était réalisée dans la communauté grâce à la collaboration avec les relais, 
dans le chef-lieu de CSCom par le personnel de santé. A l'exception de cabinet médical il existe un système de rapportage (journalier, hebdomadaire et mensuel) des données de la surveillance. Les supports de collecte (fiche de notification, registres les rapports hebdomadaires, mensuels et trimestriels) sont présents et renseignés sur quelques années au CSCom au Cs réf et la preuve de leurs transmissions au niveau supérieur est vérifiable. Au Csréf, le chargé surveillance assure le suivi et la mise en œuvre des activités de surveillance de la rougeole dans le district. Les Directives Techniques Nationales (DTN) de la rougeole sont présentes dans toutes les structures sanitaires de l'aire (Cs réf, CSCom Central et la Clinique). A l'exception du CS réf, le guide SMIR est absent au cabinet médical et au CSCom Central.

La surveillance de la rougeole n'était pas organisée au cabinet médical privé (tableau I). Les agents du cabinet médical n'étaient pas impliqués dans la surveillance de la rougeole (tableau I). Seul le Csréf a notifié un cas de rougeole au compte de l'aire de Santé de Tominian Central (tableau I). Tous les agents de santé interrogés connaissaient la rougeole (tableau III). Beaucoup de difficultés ont été signalé par les administrateurs de la santé (tableau IV).

Utilité : La surveillance épidémiologique est effectuée régulièrement dans l'aire de santé de Tominian Central de façon générale mais celle liée à la rougeole demeure timide. Aucune investigation de rougeole n'a été réalisée dans l'aire de santé de Tominian Central (tableau V).

Réactivité : Aucune notification n'a été réalisée pour la période.

Simplicité : La présence de définition de cas au CSCom et la clinique privée. Le rapportage des données issues de la surveillance sont intégrer à d'autres rapports (rapport hebdomadaire de surveillance, rapport mensuel d'activité). Les rapports hebdomadaires sont envoyés par message téléphonique, courrier en papier ou email. Quant au rapport mensuel, il est envoyé en copie dure et saisie dans le District Health Information Software version 2 (DHIS2). Le personnel du CSCom et de la clinique chargée de la notification de cas de rougeole ne sont pas ceux en charge de faire l'investigation, l'analyse et la prise de décision.

Sensibilité : Durant les dix dernières années, un seul cas suspect de rougeole (non confirmé biologiquement) a été notifié par le CSRéf pour le compte de l'aire de santé du CSCom Central. L'aire de santé de Tominian central n'a fait aucune notification durant la période étudiée.

Représentativité : L'administration du questionnaire et la revue documentaire n'ont trouvés au CSCom et à la clinique aucune notification de rougeole au cours des dix dernières années. A l'apposer, le CSRéf compte un cas pour l'aire de santé de Tominian Central enregistré au mois de Mai 2018 chez une fille d'un an. La surveillance de la rougeole n'est pas représentative dans l'aire de santé de Tominian Central.

Qualité des données :
Durant toute la période qui a concerné notre étude, la seule notification issue de l'aire de santé de Tominian Central n'était pas enregistrée sur les supports du CSCom Central encore moins sur ceux de la clinique. Les informations recueillies sur le cas notifié au CSRéf sont identique à ceux retrouvés au niveau régional en temps-personne-lieu.

\section{Discussion :}

Le but de l'évaluation des systèmes de surveillance de santé publique était de faire en sorte que les problèmes de santé soient suivis de façon efficace et efficiente (8). L'objectif de notre étude qui s'est déroulée du 1er au 30 Juin était d'évaluer le système de surveillance de la rougeole de l'aire de santé de Tominian Central sur la période de 2009-2018.

La non-participation du cabinet médical privé aux activités de surveillance épidémiologique de la rougeole contribuant ainsi à la sous notification pourrait s'expliquer par le manque de formation du personnel sur la surveillance épidémiologique (1). Les faibles couvertures vaccinales en vaccin anti rougeoleux associés à l'absence d'actions de santé publique entreprise dans l'aire de santé dans le cadre des activités de surveillance de la rougeole pourraient s'expliquer par la baisse de vigilance. II est à retenir que l'élimination de la rougeole sera rendu possible seulement si tous les enfants, où qu'ils soient, peuvent être vaccinés contre cette maladie (9). Le fait que le personnel du CSCom et du cabinet médical chargé de la notification des cas de rougeole ne soient pas ceux en charge de faire l'investigation, l'analyse et la prise de décision s'expliquerait par leur proximité avec le CSRéf et leur faible niveau d'engagement. Le CSRéf se substituerait au CSCom pour ce genre d'activités vu sa proximité. La notification d'un cas de rougeole par le CSRéf au compte de l'aire de santé de Tominian Central prouve que des cas non notifiés sont dans la communauté selon le guide le guide SMIR (3). L'aire de santé de Tominian central n'avait aucune donnée de surveillance de la rougeole pourtant, le succès de la surveillance des maladies évitables par la vaccination repose sur des données de grande qualité, y compris le lien entre les données épidémiologiques et les résultats de laboratoire (7).

\section{Conclusion :}

A l'issue de notre évaluation, nous avons constaté que l'aire de santé de Tominian Central avait déjà un système de surveillance épidémiologique opérationnel avec des insuffisances notoires par rapport à la surveillance de la rougeole (moins sensible, moins représentatif et moins réactif). Toutefois, il peut être amélioré et rendu plus efficace au prix d'un investissement supplémentaire raisonnable tout en restant acceptable par les acteurs pour surveiller d'avantage cette maladie et veiller à une gestion adéquate des données de surveillance.

\section{Références bibliographiques}


1. Ministère de la santé du Mali. Plan stratégique national d'élimination de la rougeole au Mali (2013-2020). 2013.

2. Organisation mondiale de la santé. OMS | Nouvelles données de surveillance de la rougeole pour 2019 [Internet]. WHO. 2019 [cité 19 juill 2019]. Disponible sur: http://www.who.int/immunization/newsroom/measlesdata-2019/fr/

3. Ministère de la santé du Mali M de la S du Mali. Guide Technique pour la Surveillance Intégrée de la Maladie et la Riposte au Mali. Ministère de la Santé du Mali; 2017.

4. OOAS.

OOAS_Bulletin_Epidemio_22_04_2019.pdf [Internet]. 2019. Disponible sur: http://www.sante.gov.ml/index.php/epidemie/bulletinninternational/item/3301-ooas-bulletin-d-informationsepidemiologique-de-la-15eme-semaine-2019

5. Cellule de Planification et de Statistique du Secteur Santé, Développement Social et Promotion de la Famille (CPS/SS-SD-PF). Annuaire Statistique du Système National d'Information Saniataire 2018 du Mali. Ministère de la Santéet de l'Hygiène Publique du Mali; 2019.

6. Ministère de la Santé $M$ de la $S$. Annuaire Statistique 2018 de la region de Ségou. Direction Régionale de la Santé de Ségou; 2019.

7. Organisation mondiale de la santé. Introduction de principes de surveillance des maladies évitables par la vaccination [Internet]. 2018 [cité 18 juill 2019]. Disponible sur: https://www.who.int/immunization/monitoring_surveillanc e/burden/vpd/WHO_SurveillanceVaccinePreventable_01 _Overview_French_R1.pdf

8. Jeffrey P. K, Stephen B. T, Daniel M. S, Helene D. G, Robert S. J, Suzanne B, et al. CDC Surveillance Evaluation Guideline FRENCH VERSION.pdf. Centre pour le Contrôle et Prévention des Maladies (CDC) Atlanta GA 30333; 2001.

9. Organisation mondiale de la santé. OMS | Les décès dus à la rougeole diminuent considérablement mais cette maladie tue encore 90000 personnes par an [Internet]. WHO. 2017 [cité 19 juill 2019]. Disponible sur: http://www.who.int/mediacentre/news/releases/2017/decli ne-measles-death/fr/

10. Sniadack DH, Crowcroft NS, Durrheim DN, Rota PA. Roadmap to elimination- standard measles and rubella surveillance. Wkly Epidemiol Rec. 2017;20.

Liste des tableaux :

Tableau I : Organisation de la surveillance de la rougeole, aire de Tominian Central, 2009-2018.

\begin{tabular}{lllll}
\hline \hline $\begin{array}{l}\text { Structures de } \\
\text { santé }\end{array}$ & $\begin{array}{l}\text { Appartenance à un comité } \\
\text { de surveillance } \\
\text { épidémiologique }\end{array}$ & $\begin{array}{l}\text { Archivages des } \\
\text { données de } \\
\text { surveillance }\end{array}$ & $\begin{array}{l}\text { Existence de } \\
\text { définition de cas } \\
\text { affiché }\end{array}$ & $\begin{array}{l}\text { Existence de } \\
\text { guide SMIR }\end{array}$ \\
\hline \hline CSCom Central & Non & Oui & Oui & Oui \\
Cabinet Médical & Non & Non & Oui & Non \\
CSRéf & Oui & Oui & Oui & Oui \\
\hline \hline
\end{tabular}


Tableau II : Degré d'implication des agents de santé dans la surveillance de la rougeole, Tominian

\begin{tabular}{llll}
\hline \hline Items & $\begin{array}{l}\text { Nbre de pers } \\
\text { Cscom central }\end{array}$ & $\begin{array}{l}\text { Nbre de pers } \\
\text { Cabinet Médical }\end{array}$ & $\begin{array}{l}\text { Nbre de pers } \\
\text { Csréf }\end{array}$ \\
\hline \hline $\begin{array}{l}\text { Agent dédié à la surveillance } \\
\begin{array}{l}\text { Formation des agents impliqués dans la } \\
\text { surveillance sur le guide SMIR }\end{array}\end{array}$ & 02 & 00 & 03 \\
\hline \hline
\end{tabular}

Tableau III : Connaissances des agents de santé interrogés sur la rougeole, Tominian $(\mathrm{n}=5)$

\begin{tabular}{llll}
\hline \hline Items & $\begin{array}{l}\text { Nbre de pers } \\
\text { Cscom Central }\end{array}$ & $\begin{array}{l}\text { Nbre de pers } \\
\text { Cabinet Médical }\end{array}$ & $\begin{array}{l}\text { Nombre pers } \\
\text { Csréf }\end{array}$ \\
\hline \hline Connaissance de la définition de cas & 02 & 02 & 01 \\
Simplicité de la définition de cas & 02 & 02 & 01 \\
Maladie à déclaration obligatoire & 02 & 02 & 01 \\
\hline \hline
\end{tabular}

Tableau IV : Difficultés liées à la surveillance de la rougeole, Tominian, 2009-2018.

\begin{tabular}{|c|c|c|}
\hline Difficultés & Administrateur Csréf & Administrateur DRS \\
\hline Insuffisance de ressources humaines formées & Oui & Oui \\
\hline Sous notification des cas & Non & Oui \\
\hline Résultats de laboratoire non disponibles à temps & Oui & Oui \\
\hline $\begin{array}{l}\text { Difficultés dans la mobilisation des fonds (épidémies et } \\
\text { catastrophes) }\end{array}$ & Oui & Oui \\
\hline $\begin{array}{l}\text { Insuffisance des stocks de médicaments (épidémies et } \\
\text { catastrophes) }\end{array}$ & Oui & Oui \\
\hline Insécurité & Oui & Oui \\
\hline Mécanisme de transport des échantillons trop compliqué & Oui & Oui \\
\hline
\end{tabular}

Tableau V : Point de vue des administrateurs de la santé sur la survenue d'épidémie à Tominian, 2009-2018 n=2 (Csréf et DRS)

\begin{tabular}{lcc}
\hline \hline Items & Administrateur Csréf & Administrateur DRS \\
\hline \hline $\begin{array}{l}\text { Nombre d'épidémies de rougeole survenues dans le } \\
\text { district sanitaire de Tominian }\end{array}$ & 3 & 3 \\
$\begin{array}{l}\text { Cas de rougeole notifiés dans l'aire de santé de T. } \\
\text { Central }\end{array}$ & 1 & 1 \\
$\begin{array}{l}\text { Nombre d'épidémies de rougeole survenue dans l'aire de } \\
\text { santé de T. Central } \\
\text { Investigation menée dans l'aire de T. Central }\end{array}$ & 0 & 0 \\
\hline \hline
\end{tabular}

